\title{
The low-bias conducting mechanism of single-molecule junctions constructed with methylsulfide linker groups and gold electrodes
}

Minglang Wang, Yongfeng Wang, Stefano Sanvito, and Shimin Hou

Citation: The Journal of Chemical Physics 147, 054702 (2017);

View online: https://doi.org/10.1063/1.4996745

View Table of Contents: http://aip.scitation.org/toc/jcp/147/5

Published by the American Institute of Physics

\section{Articles you may be interested in}

Temperature dependent tunneling conductance of single molecule junctions

The Journal of Chemical Physics 146, 092311 (2017); 10.1063/1.4973318

Uncovering hierarchical data structure in single molecule transport

The Journal of Chemical Physics 146, 092321 (2017); 10.1063/1.4974937

Enhancing the conductivity of molecular electronic devices

The Journal of Chemical Physics 146, 092310 (2016); 10.1063/1.4972992

Adsorption and charge transfer interactions of bi-isonicotinic acid on $\mathrm{Ag}(111)$

The Journal of Chemical Physics 147, 054703 (2017); 10.1063/1.4996746

Destructive quantum interference in electron transport: A reconciliation of the molecular orbital and the atomic orbital perspective

The Journal of Chemical Physics 146, 092308 (2016); 10.1063/1.4972572

Transport mirages in single-molecule devices

The Journal of Chemical Physics 146, 092330 (2017); 10.1063/1.4975767

\section{AIP | The Jounal of Chemical Physics}




\title{
The low-bias conducting mechanism of single-molecule junctions constructed with methylsulfide linker groups and gold electrodes
}

\author{
Minglang Wang, ${ }^{1}$ Yongfeng Wang, ${ }^{1,2}$ Stefano Sanvito, ${ }^{3}$ and Shimin $\mathrm{Hou}^{1,2, \mathrm{a})}$ \\ ${ }^{1}$ Key Laboratory for the Physics and Chemistry of Nanodevices, Department of Electronics, Peking University, \\ Beijing 100871, China \\ ${ }^{2}$ Beida Information Research (BIR), Tianjin 300457, China \\ ${ }^{3}$ School of Physics, AMBER and CRANN Institute, Trinity College, Dublin 2, Ireland
}

(Received 26 April 2017; accepted 18 July 2017; published online 2 August 2017)

\begin{abstract}
The atomic structure and electronic transport properties of two types of molecular junctions, in which a series of saturated and conjugated molecules are symmetrically connected to gold electrodes through methylsulfide groups, are investigated using the non-equilibrium Green's function formalism combined with density functional theory. Our calculations show that the low-bias junction conductance is determined by the electronic tunneling between the two $\mathrm{Au}-\mathrm{S}$ donor-acceptor bonds formed at the molecule-electrode interfaces. For alkanes with 4, 6, and 8 carbon atoms in the chain, the Au-S bonds moderately couple with the $\sigma$-type frontier molecular orbitals of the alkane backbone and thus prefer to be coplanar with the alkane backbone in the junction. This results in an exponential decrease of the junction conductance as a function of the number of methylene groups. In contrast, the Au-S bonds couple strongly with the $\pi$-type orbitals of the 1,4'-bis(methylsulfide)benzene and 4,4'-bis(methylsulfide)biphenyl molecules and thus tend to be perpendicular to the neighboring benzene rings, leading to the rather large junction conductance. Our findings contribute to the understanding of the low-bias conducting mechanism and facilitate the design of molecular electronic devices with methylsulfide groups and gold electrodes. Published by AIP Publishing. [http://dx.doi.org/10.1063/1.4996745]
\end{abstract}

\section{INTRODUCTION}

Exploring the structure-property relationship of electronic devices using single molecules as the active component is the cutting edge research in molecular electronics. ${ }^{1}$ Singlemolecule devices also provide an ideal platform for the investigation of basic quantum transport concepts. When a single molecule is wired between two metal electrodes, the moleculeelectrode contacts that are formed by the metal electrodes and the linker groups flanked at the two ends of the central molecule have profound effects on the electronic transport properties of molecular junctions. ${ }^{2}$ Besides the widely used thiol, pyridine, and amine groups, ${ }^{3-5}$ methylsulfide (SMe) is often employed as the linker bonded to gold electrodes. ${ }^{6-33}$ For example, with the Au-SMe bonds single-molecule diodes with rectification ratios higher than 200 at voltages as low as $370 \mathrm{mV}$ were fabricated in an ionic solution. ${ }^{27}$ Furthermore, single-molecule switches operating through a stereoelectronic effect were first realized in oligosilane molecules connected to gold electrodes through SMe linkers. ${ }^{25}$ It is generally accepted that for thiol groups the $\mathrm{Au}-\mathrm{S}$ covalent bonds are formed at the molecule-electrode interfaces while pyridine, amine, and methylsulfide groups form donor-acceptor bonds with undercoordinated gold atoms on the electrode surface because of the lone pairs in the corresponding $\mathrm{N}$ or $\mathrm{S}$ atom. Unfortunately, up to now the detailed atomic structures of the

a) Author to whom correspondence should be addressed: smhou@pku.edu.cn molecule-electrode contacts are still not clearly known and thus out of control in experiments. In contrast, computer simulations can take into account the atomic details of molecular junctions and improve our understanding of their electronic transport properties. Although many theoretical investigations have been devoted to exploring the conducting mechanism of molecular junctions constructed using the thiol, pyridine, and amine groups, ${ }^{34-40}$ only a few theoretical studies focus on the intrinsic properties of the molecule-electrode interfaces formed by the Au-SMe bonds. ${ }^{31,41}$ Therefore, it is highly desirable to carry out first-principles calculations on representative molecular junctions constructed with the $\mathrm{Au}-\mathrm{SMe}$ bonds.

Here we investigate the structural and electronic transport properties of a series of saturated and conjugated molecules with SMe groups at the two ends. This is realized by employing the non-equilibrium Green's function formalism (NEGF) combined with density function theory (DFT), i.e., the NEGF + DFT approach. ${ }^{42-50}$ Our calculations show that the Au$\mathrm{S}$ donor-acceptor bonds formed by the SMe group with the $\mathrm{Au}$ adatom on the electrode surface play an important role in the low-bias junction conductance. When the $\mathrm{Au}-\mathrm{S}$ bonds are nearly coplanar with the alkane backbones or perpendicular to the adjacent benzene rings in the Au-molecule-Au junctions, the calculated values of the low-bias junction conductance are in semi-quantitative or even quantitative agreement with the measured values. In this case the transport is dominated by the highest occupied molecular orbital (HOMO) and the HOMO-1 orbital of the central molecule. 


\section{CALCULATION METHOD}

We employ the SIESTA software package to investigate the atomic and electronic structures of Au-molecule-Au molecular junctions and the SMEAGOL code to study their electronic transport properties. ${ }^{49-51}$ SIESTA is an efficient DFT package for numerical simulations of large system with affordable resources, in which the wave functions of valence electrons are expanded over a finite-range numerical basis set and the core electrons are described by norm-conserving Troullier-Martins pseudopotentials. ${ }^{51,52}$ While a double-zeta plus polarization (DZP) basis set is used for $\mathrm{H}, \mathrm{C}$, and $\mathrm{S}$ atoms, two different types of basis functions are used for $\mathrm{Au}$, respectively, in the bulk and at the surface. In more detail, a DZP basis is used for the Au surface atoms, while a singlezeta plus polarization (SZP) basis is used for the bulk. The exchange-correlation functional is treated at the level of the generalized gradient approximation (GGA) within the PerdewBurke-Ernzerhof (PBE) formulation. ${ }^{53}$ Geometry optimization is performed by standard conjugate gradient until all the atomic forces are smaller than $0.03 \mathrm{eV} \AA^{-1}$.

SMEAGOL is a practical implementation of the NEGF +DFT approach, which uses SIESTA as the DFT platform. ${ }^{49,50}$ Periodic boundary conditions are applied in the plane transverse to the transport direction, while the unit cell of the extended molecule includes the central molecule and ten atomic layers of the gold electrodes with a $(3 \times 3)$ supercell. An equivalent cutoff of $200.0 \mathrm{Ry}$ is taken for the real space grid integration. The charge density is integrated over 24 energy points along the semi-circle, 24 points along the line in the complex plane, while 24 poles are used for the Fermi function (the electronic temperature is $25 \mathrm{meV}$ ). The transmission coefficient $T(E)$ of the molecular junction is evaluated as

$$
T(E)=\frac{1}{\Omega_{2 D B Z}} \int_{2 D B Z} T(\vec{k} ; E) d \vec{k},
$$

where $\Omega_{2 \mathrm{DBZ}}$ is the area of the two-dimensional Brillouin zone (2DBZ) in the transverse directions. The $k$-dependent transmission coefficient $T(\vec{k} ; E)$ is obtained as

$$
T(\vec{k} ; E)=\operatorname{Tr}\left[\Gamma_{L} G_{M}^{R} \Gamma_{R} G_{M}^{R+}\right],
$$

where $G_{M}^{R}$ is the retarded Green's function matrix of the extended molecule and $\Gamma_{\mathrm{L}}\left(\Gamma_{\mathrm{R}}\right)$ is the broadening function matrix describing the interaction of the extended molecule with the left-hand (right-hand) side electrode. Here, we calculate the transmission coefficient by sampling $4 \times 4$ k-points in the transverse 2DBZ.

\section{RESULTS AND DISCUSSION}

We start our studies from the investigation of the atomic structure and the electronic transport properties of alkanes with 4,6 , and 8 methylene groups terminated by two SMe groups. The ground state of the isolated 1,4-bis(methylsulfide)butane $\left(\mathrm{SMe}-\mathrm{C}_{4}-\mathrm{SMe}\right)$ molecule has the two carbon atoms in the SMe groups optimized to be out of the plane defined by the four carbon atoms of the butane backbone [Fig. 1(a)], which is characterized by the dihedral angle $\angle \mathrm{C}(\mathrm{SMe})-\mathrm{S}-\mathrm{C}-\mathrm{C}=71.6^{\circ}$. Both the HOMO and HOMO-1 orbitals are dominated by

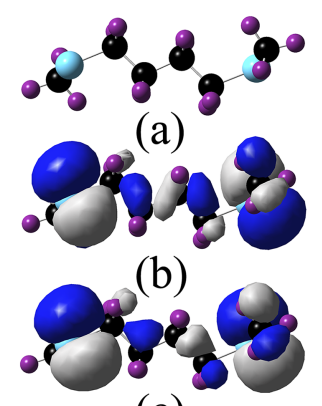

(c)

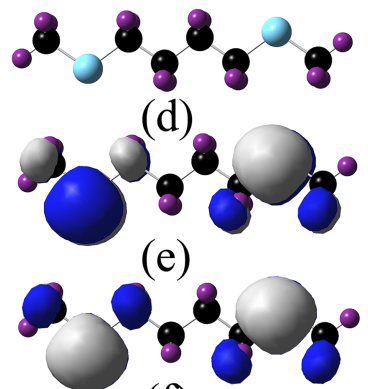

(f)
FIG. 1. The optimized atomic structure (a), HOMO (b), and HOMO-1 (c) of the $\mathrm{SMe}-\mathrm{C}_{4}-\mathrm{SMe}$ molecule with the two carbon atoms in the $\mathrm{SMe}$ groups out of the plane of the butane backbone; the optimized atomic structure (d), HOMO (e), and HOMO-1 (f) of the SMe- $\mathrm{C}_{4}-\mathrm{SMe}$ molecule with the two carbon atoms in the SMe groups coplanar with the butane backbone. Purple, black, and cyan spheres are, respectively, hydrogen, carbon, and sulfur atoms.

the occupied S 3p atomic orbitals in the two SMe groups. Since these two S $3 p$ atomic orbitals are coplanar with the four carbon atoms of the butane backbone, they are coupled across the butane backbone resulting in an energy splitting of $\sim 130 \mathrm{meV}$ between the HOMO and HOMO-1 orbitals. It should be noted that the $\mathrm{SMe}-\mathrm{C}_{4}-\mathrm{SMe}$ molecule with the two carbon atoms in the SMe groups coplanar with the butane backbone (the dihedral angle $\angle \mathrm{C}(\mathrm{SMe})-\mathrm{S}-\mathrm{C}-\mathrm{C}=180^{\circ}$ ) is also a local minimum, and its total energy is only $26 \mathrm{meV}$ higher than that of the ground state geometry. In contrast, the corresponding HOMO and HOMO- 1 orbitals are almost degenerate and are both composed of two localized S 3p atomic orbitals perpendicular to the plane of the butane backbone [see Figs. 1(e) and 1(f)]. Due to the rather small energy difference, the SMe$\mathrm{C}_{4}-\mathrm{SMe}$ molecule with both these two conformations may appear in $\mathrm{Au}-\mathrm{SMe}-\mathrm{C}_{4}-\mathrm{SMe}-\mathrm{Au}$ molecular junctions fabricated with the scanning tunneling microscopy break junction (STM-BJ) method at room temperature. ${ }^{6,10,28}$

Figure 2(a) shows the optimized atomic structure of the $\mathrm{Au}-\mathrm{SMe}-\mathrm{C}_{4}-\mathrm{SMe}-\mathrm{Au}$ molecular junction, in which the two $\mathrm{SMe}$ groups are assumed to bind with the Au adatoms of the $\mathrm{Au}(111)$ surface. In order to find the equilibrium structure, we systematically vary the separation between the two gold electrodes and optimize the positions of the $\mathrm{SMe}-\mathrm{C}_{4}-\mathrm{SMe}$ molecule, gold adatoms, and those at the electrode surfaces until the total energy reaches a local minimum. The bond length of the $\mathrm{Au}-\mathrm{S}$ donor-acceptor bonds is optimized to be $2.47 \AA$, and the dihedral angles $\angle \mathrm{Au}-\mathrm{S}-\mathrm{C}-\mathrm{C}$ and $\angle \mathrm{C}(\mathrm{SMe})-$ $\mathrm{S}-\mathrm{C}-\mathrm{C}$ are, respectively, optimized to be $\sim 176^{\circ}$ and $\sim 82^{\circ}$. The corresponding equilibrium transmission spectrum is presented in Fig. 2(b). Clearly, there are two transmission peaks around the Fermi level, one peak centered at $-1.77 \mathrm{eV}$ and the other centered at $2.64 \mathrm{eV}$. Because these two transmission peaks decay rapidly towards the Fermi level, the transmission coefficient at the Fermi level is calculated to be 2.8 $\times 10^{-3}$, which is consistent with the experimental value of $1.4 \times 10^{-3}{ }^{6,10}$ A deep understanding can be obtained by projecting the transmission onto the frontier molecular orbitals of the $\mathrm{SMe}-\mathrm{C}_{4}-\mathrm{SMe}$ molecule [Fig. 2(c)]. ${ }^{54}$ The transmission peak at $-1.77 \mathrm{eV}$ and the transmission around the Fermi level are dominated by the HOMO and HOMO-1 orbitals of the $\mathrm{SMe}-\mathrm{C}_{4}-\mathrm{SMe}$ molecule. By comparing with the local density 

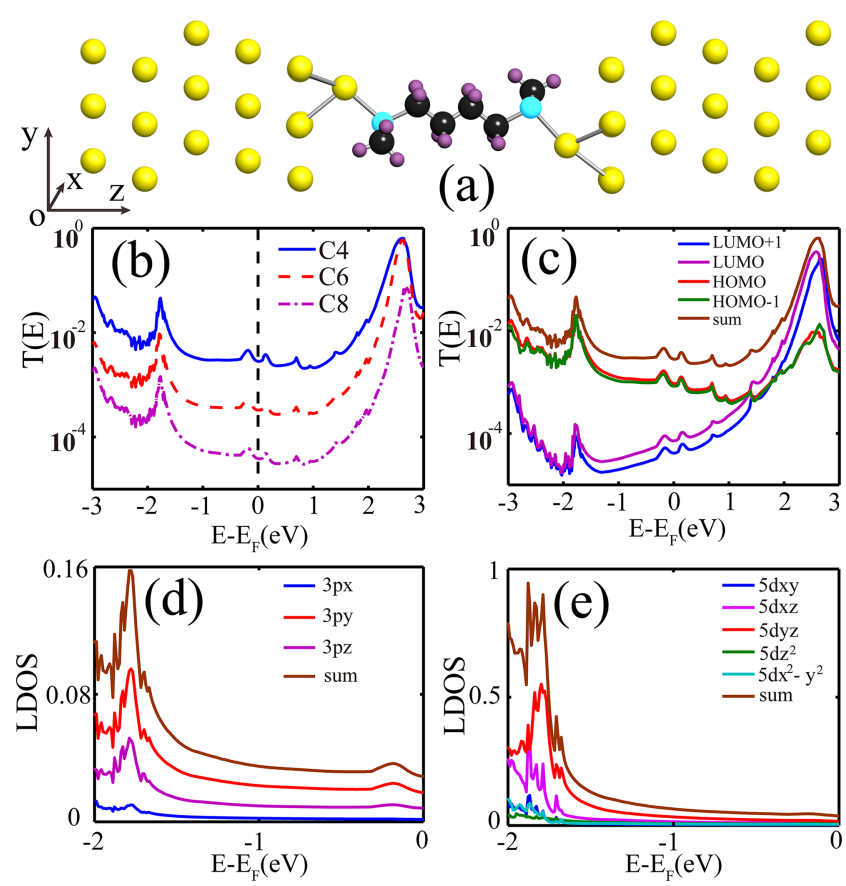

FIG. 2. The atomic structure and electronic transport properties of the Au$\mathrm{SMe}-\mathrm{C}_{4}-\mathrm{SMe}-\mathrm{Au}$ molecule junction with the $\mathrm{Au}-\mathrm{S}$ bonds nearly coplanar with the butane backbone: (a) the optimized atomic structure, (b) the equilibrium transmission spectrum, (c) the transmission spectrum projected onto frontier molecular orbitals of $\mathrm{SMe}-\mathrm{C}_{4}-\mathrm{SMe}$, (d) the LDOS projected onto the $\mathrm{S} 3 \mathrm{p}$ atomic orbitals, and (e) the LDOS projected onto the $5 \mathrm{~d}$ orbitals of the Au adatom. Purple, black, cyan, and yellow color spheres in (a) are, respectively, hydrogen, carbon, sulfur, and gold atoms. For comparison, the equilibrium transmission spectra of the $\mathrm{Au}-\mathrm{SMe}-\mathrm{C}_{6}-\mathrm{SMe}-\mathrm{Au}$ and $\mathrm{Au}-\mathrm{SMe}-\mathrm{C}_{8}-\mathrm{SMe}-\mathrm{Au}$ molecular junctions are also given in (b).

of states (LDOS) projected onto the atomic orbitals of the sulfur atoms in the SMe groups and the Au adatoms [Figs. 2(d) and 2(e)], we establish that the HOMO and HOMO-1 orbitals of the $\mathrm{SMe}-\mathrm{C}_{4}-\mathrm{SMe}$ molecule are mainly composed of the $\mathrm{S}$ $3 \mathrm{p}_{\mathrm{y}}$ atomic orbitals when the butane backbone is placed along the $\mathrm{yOz}$ plane in the junction. This in turn couples to the $\mathrm{Au}$ $5 \mathrm{~d}_{\mathrm{yz}}$ atomic orbital contributing to the transmission peak centered at $-1.77 \mathrm{eV}$ and determines the transmission around the Fermi level.

In contrast, when the two carbon atoms in the SMe groups are nearly coplanar with the butane backbone in the $\mathrm{Au}-$ $\mathrm{SMe}-\mathrm{C}_{4}-\mathrm{SMe}-\mathrm{Au}$ junction, which is characterized by the dihedral angles $\angle \mathrm{Au}-\mathrm{S}-\mathrm{C}-\mathrm{C}=87^{\circ}$ and $\angle \mathrm{C}(\mathrm{SMe})-\mathrm{S}-\mathrm{C}-\mathrm{C}$ $=170^{\circ}$ [Fig. 3(a)], the junction transmission is reduced significantly. As it can be seen in Fig. 3(b), the transmission coefficient at the Fermi level is calculated to be $4.7 \times 10^{-5}$ that is much less than the measured value, despite the fact that two transmission peaks appear, respectively, at $-1.77 \mathrm{eV}$ and $2.63 \mathrm{eV}$. The much-reduced transmission should be ascribed to the more localized HOMO and HOMO- 1 orbitals of the $\mathrm{SMe}-\mathrm{C}_{4}-\mathrm{SMe}$ molecule in this conformation [Figs. 1(e) and 1(f)], since the electronic coupling of the $\mathrm{Au}-\mathrm{S}$ interfaces should not be changed too much due to the very similar $\mathrm{Au}-\mathrm{S}$ donor-acceptor bond lengths $(2.49 \AA)$. Therefore, the most probable molecule-electrode interfaces in $\mathrm{Au}-\mathrm{SMe}-$ $\mathrm{C}_{4}-\mathrm{SMe}-\mathrm{Au}$ molecule junctions are formed by the $\mathrm{Au}-\mathrm{S}$ donor-acceptor bonds that are nearly coplanar with the butane backbone.

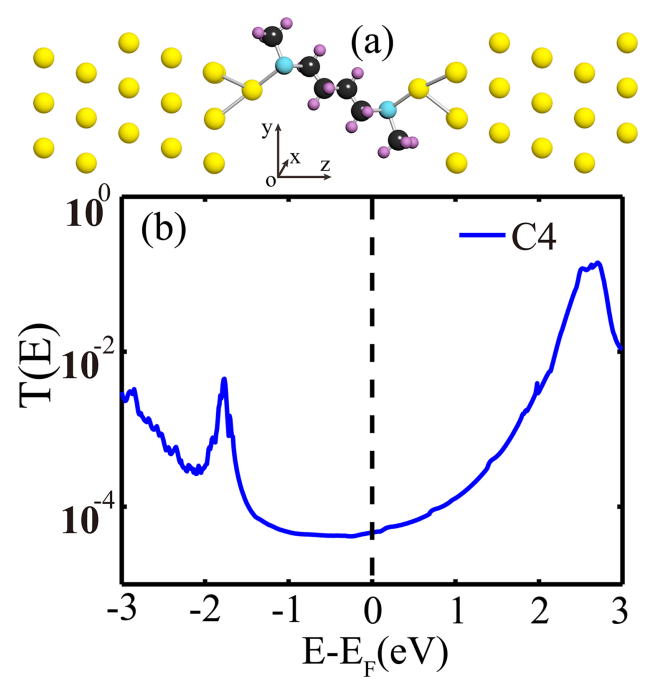

FIG. 3. The optimized atomic structure (a) and the equilibrium transmission spectrum (b) of the $\mathrm{Au}-\mathrm{SMe}-\mathrm{C}_{4}-\mathrm{SMe}-\mathrm{Au}$ molecular junction with the $\mathrm{Au}-\mathrm{S}$ bonds out of the plane of the butane backbone.

Then we calculate the transport properties of 1,6bis(methylsulfide)hexane (SMe- $\left.\mathrm{C}_{6}-\mathrm{SMe}\right)$ and 1,8-bis (methylsulfide)octane $\left(\mathrm{SMe}-\mathrm{C}_{8}-\mathrm{SMe}\right)$ molecules contacted with gold electrodes [Fig. 2(b)]. With the optimized Au-S bond length of $2.47 \AA$ and the dihedral angle $\angle \mathrm{Au}-\mathrm{S}-\mathrm{C}-\mathrm{C}$ approaching $180^{\circ}$, the transmission coefficients at the Fermi level are calculated to be $3.3 \times 10^{-4}$ for $\mathrm{Au}-\mathrm{SMe}-\mathrm{C}_{6}-\mathrm{SMe}-\mathrm{Au}$ and 3.8 $\times 10^{-5}$ for $\mathrm{Au}-\mathrm{SMe}-\mathrm{C}_{8}-\mathrm{SMe}-\mathrm{Au}$ junctions, both in excellent agreement with the corresponding measured values of 3.1 $\times 10^{-4}$ and $3.9 \times 10^{-5}{ }^{6,26}$ By using the same analysis carried out for the $\mathrm{Au}-\mathrm{SMe}-\mathrm{C}_{4}-\mathrm{SMe}-\mathrm{Au}$ junction, we can confirm that the interaction between the $\mathrm{Au} 5 \mathrm{~d}_{\mathrm{yz}}$ and $\mathrm{S} 3 \mathrm{p}_{\mathrm{y}}$ atomic orbitals dominates the transmission peak centered at $-1.77 \mathrm{eV}$ and the transmission around the Fermi level for both $\mathrm{Au}-\mathrm{SMe}-\mathrm{C}_{6}-\mathrm{SMe}-\mathrm{Au}$ and $\mathrm{Au}-\mathrm{SMe}-\mathrm{C}_{8}-\mathrm{SMe}-\mathrm{Au}$. Therefore, the low-bias conductance of the $\mathrm{Au}-\mathrm{SMe}-\mathrm{C}_{n}-\mathrm{SMe}-\mathrm{Au}$ molecular junctions mainly originates from the electronic tunneling between the two localized $\mathrm{Au}-\mathrm{S}$ donor-acceptor bonds that are mediated by the central alkane chains, where $n$ is the number of carbon atoms in the chain. The longer the alkane chains and the larger the dihedral angle between the $\mathrm{Au}-\mathrm{S}$ bond and the alkane backbone, the lower the junction conductance. This tunneling mechanism of conduction is also corroborated by the length-dependence of the junction conductance. For these three molecular junctions, the conductance decreases exponentially with the increase of the length of the central alkanes, which can be described with the relation $G$ $\sim \exp (-\beta n)$. Here, $G$ is the junction conductance, and $\beta$ is the decay constant. The decay constant $\beta$ is determined to be 1.0 per methylene group, in good agreement with the measured value of $0.94 \pm 0.05 .^{28}$

Next we turn to investigate the electronic transport properties of conjugated molecules terminated with one $\mathrm{SMe}$ group at each end, and choose 1,4'-bis(methylsulfide)benzene (SMe-Ph-SMe) and 4,4'-bis(methylsulfide)biphenyl (SMe$\mathrm{Ph}_{2}-\mathrm{SMe}$ ) as the representative cases. Different from the $\mathrm{SMe}-\mathrm{C}_{n}-\mathrm{SMe}$ alkanes investigated above, in the ground state the two carbon atoms in the SMe groups are found to be 


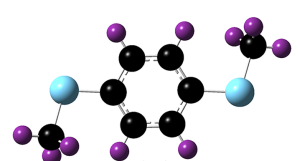

(a)

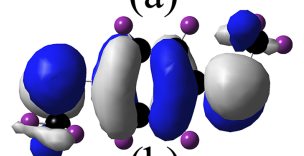

(b)

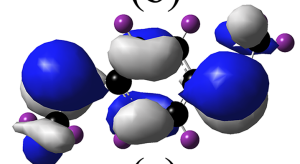

(c)

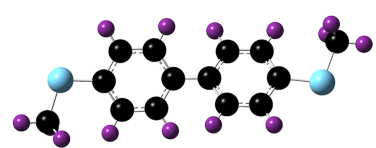

(d)

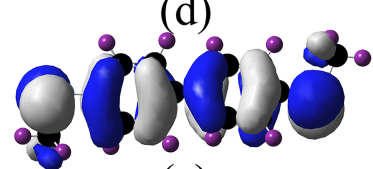

(e)

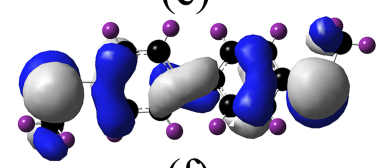

(f)
FIG. 4. The optimized atomic structure (a), HOMO (b), and HOMO-1 (c) of the $\mathrm{SMe}-\mathrm{Ph}-\mathrm{SMe}$ molecule; the optimized atomic structure (d), HOMO (e), and HOMO-1 (f) of the $\mathrm{SMe}-\mathrm{Ph}_{2}-\mathrm{SMe}$ molecule.

coplanar with the benzene backbone for the isolated $\mathrm{SMe}-$ $\mathrm{Ph}-\mathrm{SMe}$ molecule [Fig. 4(a)]. Thus, the S 3p lone pairs in the two SMe groups can have a strong coupling with the $\pi$-type orbitals of the benzene backbone. The S-C(benzene) bond lengths are optimized to be $1.777 \AA$ shorter than the $\mathrm{S}-\mathrm{C}$ (methylene) bond with a length of $1.836 \AA$ in the SMe$\mathrm{C}_{4}-\mathrm{SMe}$ molecule, further strengthening the coupling between the $S 3 p$ lone pairs and the $\pi$-type benzene orbitals. As shown in Figs. 4(b) and 4(c), the HOMO and HOMO-1 orbitals of the $\mathrm{SMe}-\mathrm{Ph}-\mathrm{SMe}$ molecule are both $\pi$-type delocalized along the entire molecule including the benzene backbone and the two sulfur atoms of the SMe groups, and their energy splitting is about $1.15 \mathrm{eV}$. Similar atomic and electronic structures are obtained for the isolated $\mathrm{SMe}-\mathrm{Ph}_{2}-\mathrm{SMe}$ molecule [Figs. 4(d)4(f)]. The carbon atoms in the two SMe groups are coplanar with their neighboring benzene rings; the HOMO and HOMO1 orbitals are also $\pi$-type but with a smaller energy splitting of $0.58 \mathrm{eV}$.

For the Au-SMe-Ph-SMe-Au molecular junction [Fig. 5(a)], the two SMe groups are also assumed to bind with the $\mathrm{Au}$ adatom of the $\mathrm{Au}(111)$ surface with an optimized $\mathrm{Au}-$ $\mathrm{S}$ bond length of $2.51 \AA$ and a dihedral angle $\angle \mathrm{Au}-\mathrm{S}-\mathrm{C}-\mathrm{C}$

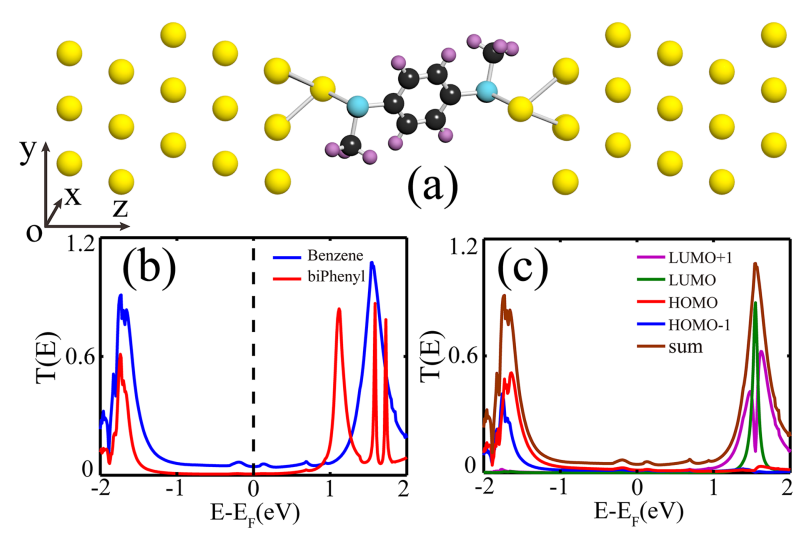

FIG. 5. The atomic structure and electronic transport properties of the $\mathrm{Au}-$ $\mathrm{SMe}-\mathrm{Ph}-\mathrm{SMe}-\mathrm{Au}$ molecule junction: (a) the optimized atomic structure, (b) the equilibrium transmission spectrum, and (c) the transmission spectrum projected onto frontier molecular orbitals of $\mathrm{SMe}-\mathrm{Ph}-\mathrm{SMe}$. For comparison, the equilibrium transmission spectra of the $\mathrm{Au}-\mathrm{SMe}-\mathrm{Ph}_{2}-\mathrm{SMe}-\mathrm{Au}$ molecular junctions are also given in (b). of $96^{\circ}$. The equilibrium transmission spectrum is shown in Fig. 5(b). The first transmission peak below the Fermi level is located at $-1.73 \mathrm{eV}$, indicating that the $\mathrm{Au}-\mathrm{S}$ bonding is almost the same as that in the $\mathrm{Au}-\mathrm{SMe}-\mathrm{C}_{n}-\mathrm{SMe}-\mathrm{Au}$ junctions. In contrast, the first transmission peak above the Fermi level is shifted downwards in energy to $1.55 \mathrm{eV}$ due to the much reduced HOMO-LUMO gap of the SMe-Ph-SMe molecule. Here, LUMO is the acronym of the lowest unoccupied molecular orbital. By projecting the transmission spectrum onto the frontier molecular orbitals of SMe-Ph-SMe [Fig. 5(c)], we can deduce that the transmission peak at $-1.73 \mathrm{eV}$ and the transmission around the Fermi level are dominated by the HOMO and HOMO-1 orbitals. The transmission coefficient at the Fermi level is calculated to be $4.3 \times 10^{-2}$, slightly larger than the actual measured value of $(0.9 \pm 0.05) \times 10^{-2} .8$ Considering that GGA functionals like PBE always underestimate the HOMO position (too high in energy) and thus overestimate the low-bias junction conductance, ${ }^{55}$ this result is quite acceptable. Since the $\pi$-type HOMO and HOMO-1 orbitals of $\mathrm{SMe}-\mathrm{Ph}-\mathrm{SMe}$ provide a better coupling between the $\mathrm{Au}-\mathrm{S}$ donor-acceptor bonds, the $\mathrm{Au}-\mathrm{SMe}-\mathrm{Ph}-\mathrm{SMe}-\mathrm{Au}$ junction is more conducting than the $\mathrm{Au}-\mathrm{SMe}-\mathrm{C}_{4}-\mathrm{SMe}-\mathrm{Au}$ one. With the optimized $\mathrm{Au}-\mathrm{S}$ bond length of $2.51 \AA$ and the dihedral angle of $\angle \mathrm{Au}-\mathrm{S}-\mathrm{C}-\mathrm{C}=66^{\circ}$, the calculated transmission spectrum of the $\mathrm{Au}-\mathrm{SMe}-\mathrm{Ph}_{2}-\mathrm{SMe}-\mathrm{Au}$ junction is presented in Fig. 5(b). When compared to that of the $\mathrm{Au}-$ $\mathrm{SMe}-\mathrm{Ph}-\mathrm{SMe}-\mathrm{Au}$ junction, the first transmission peak below the Fermi level does not change its position but presents a reduced amplitude. The transmission coefficient at the Fermi level is only $6.2 \times 10^{-3}$, in good agreement with the measured value of $2 \times 10^{-3},{ }^{17}$ though the first transmission peak above the Fermi level is further shifted downwards to $1.12 \mathrm{eV}$. The transmission around the Fermi level is still mainly contributed by the HOMO and HOMO- 1 orbitals of the $\mathrm{SMe}-\mathrm{Ph}_{2}-\mathrm{SMe}$ molecule. Although the $\mathrm{SMe}-\mathrm{Ph}_{2}-\mathrm{SMe}$ molecule has a higher conjugation than that of $\mathrm{SMe}-\mathrm{Ph}-\mathrm{SMe}$ and thus has a smaller HOMO-LUMO gap, the dihedral angle $\left(\sim 36^{\circ}\right)$ between two benzene rings reduces the efficiency of the electronic coupling between the $\mathrm{S} 3 \mathrm{p}$ lone pairs of the two SMe groups leading to the lower conductance of the $\mathrm{Au}-\mathrm{SMe}-\mathrm{Ph}_{2}-\mathrm{SMe}-\mathrm{Au}$ junction.

Transport calculations have also been performed for junctions under moderate compressive and tensile strain, obtained by changing the distance between the electrodes up to $\pm 0.5 \AA$ from the equilibrium position. In all cases, although the relaxation gives alterations in the details of the bonding structure, we find that the transmission remains almost unchanged, indicating that the SMe anchoring group makes the transport stable against geometry fluctuations.

\section{CONCLUSION}

We have investigated the atomic structures and electronic transport properties of molecular junctions employing the NEGF + DFT approach, in which the alkanes and aromatic molecules are connected to gold electrodes through SMe linkers. Our calculations show that electronic tunneling between the $\mathrm{Au}-\mathrm{S}$ bonding states at the two molecule-electrode interfaces dominates the low-bias junction conductance, which is 
mediated by the central aliphatic and aromatic molecules. Since the frontier molecular orbitals of the central aliphatic and aromatic molecules are, respectively, $\sigma$-type and $\pi$-type, the $\mathrm{Au}-\mathrm{S}$ donor-acceptor bonds tend to be parallel to the alkane backbones or be perpendicular to the neighboring benzene rings, thus enabling a better electronic coupling between the $\mathrm{Au}-\mathrm{S}$ bonding states. This is also the main reason for the low-bias conductance of the $\mathrm{Au}-\mathrm{SMe}-\mathrm{Ph}-\mathrm{SMe}-\mathrm{Au}$ and $\mathrm{Au}-$ $\mathrm{SMe}-\mathrm{Ph}_{2}-\mathrm{SMe}-\mathrm{Au}$ junctions to be much larger than those of the $\mathrm{Au}-\mathrm{SMe}-\mathrm{C}_{n}-\mathrm{SMe}-\mathrm{Au}$ junctions with a similar molecular length. Our findings are helpful in the development and application of methyl sulfide linker groups in future molecular electronic devices.

\section{ACKNOWLEDGMENTS}

This project was supported by the National Natural Science Foundation of China (Grant Nos. 61671021 and 61621061), the MOST of China (Grant No. 2013CB933404), and the National Key Research \& Development Program (Grant No. 2016YFA0201901). S.S. thanks additional funding support from the European Research Council (QUEST project) and by AMBER (No. 12/RC/2278).

${ }^{1}$ D. Xiang, X. Guo, and T. Lee, Chem. Rev. 116, 4318 (2016).

${ }^{2}$ E. Leary, A. La Rosa, M. Teresa González, G. Rubio-Bollinger, N. Agraït, and N. Marth, Chem. Soc. Rev. 44, 920 (2015).

${ }^{3}$ M. A. Reed, C. Zhou, C. J. Muller, T. P. Burgin, and J. M. Tour, Science 278, 252 (1997).

${ }^{4}$ B. Q. Xu and N. J. Tao, Science 301, 1221 (2003).

${ }^{5}$ L. Venkataraman, J. E. Klare, C. Nuckolls, M. S. Hybertsen, and M. L. Steigerwald, Nature 442, 904 (2006).

${ }^{6}$ Y. S. Park, A. C. Whalley, M. Kamenetska, M. L. Steigerwald, M. S. Hybertsen, C. Nuckolls, and L. Venkataraman, J. Am. Chem. Soc. 129, 15768 (2007).

${ }^{7}$ M. Kamenetska, M. Koentopp, A. C. Whalley, Y. S. Park, M. L. Steigerwald, C. Nuckolls, M. S. Hybertsen, and L. Venkataraman, Phys. Rev. Lett. 102, 126803 (2009).

${ }^{8}$ Y. S. Park, J. R. Widawsky, M. Kamenetska, M. L. Steigerwald, M. S. Hybertsen, C. Nuckolls, and L. Venkataraman, J. Am. Chem. Soc. 131, 10820 (2009).

${ }^{9}$ J. S. Meisner, M. Kamenetska, M. Krikorian, M. L. Steigerwald, L. Venkataraman, and C. Nuckolls, Nano Lett. 11, 1575 (2011).

${ }^{10}$ M. Frei, S. V. Aradhya, M. S. hybertsen, and L. Venkataraman, J. Am. Chem. Soc. 134, 4003 (2012).

${ }^{11}$ R. S. Klausen, J. R. Widawsky, M. L. Steigerwald, L. Venkataraman, and C. Nuckolls, J. Am. Chem. Soc. 134, 4541 (2012).

${ }^{12}$ S. V. Aradhya, J. S. Meisner, M. Krikorian, S. Ahn, R. Parameswaran, M. L. Steigerwald, C. Nuckolls, and L. Venkataraman, Nano Lett. 12, 1643 (2012).

${ }^{13}$ S. Ahn, S. V. Aradhya, R. S. Klausen, B. Capozzi, X. Roy, M. L. Steigerwald, C. Nuckolls, and L. Venkataraman, Phys. Chem. Chem. Phys. 14, 13841 (2012).

${ }^{14}$ H. Vazquez, R. Skouta, S. Schneebeli, M. Kamenetska, R. Breslow, L. Venkataraman, and M. S. Hybertsen, Nat. Nanotechnol. 7, 663 (2012).

${ }^{15}$ X. Roy, C. L. Schenck, S. Ahn, R. A. Lalancette, L. Venkataraman, C. Nuckolls, and M. L. Steigerwald, Angew. Chem., Int. Ed. 51, 12473 (2012).

${ }^{16}$ J. S. Meisner, S. Ahn, S. V. Aradhya, M. Krikorian, R. Parameswaran, M. L. Steigerwald, L. Venkataraman, and C. Nuckolls, J. Am. Chem. Soc. 134, 20440 (2012)

${ }^{17}$ E. J. Dell, B. Capozzi, K. H. Dubay, T. C. Berkelbach, J. R. Moreno, D. R. Reichman, L. Venkataraman, and L. M. Campos, J. Am. Chem. Soc. 135, 11724 (2013).
${ }^{18}$ A. Batra, P. Darancet, Q. Chen, J. S. Meisner, J. R. Widawsky, J. B. Neaton, C. Nuckolls, and L. Venkataraman, Nano Lett. 13, 6233 (2013).

${ }^{19}$ T. A. Su, J. R. Widawsky, H. Li, R. S. Klausen, J. L. Leighton, M. L. Steigerwald, L. Venkataraman, and C. Nuckolls, J. Am. Chem. Soc. 135, 18331 (2013).

${ }^{20}$ A. Batra, J. S. Meisner, P. Darancet, Q. Chen, M. L. Steigerwald, C. Nuckolls, and L. Venkataraman, Faraday Discuss. 174, 79 (2014).

${ }^{21}$ S. V. Aradhya, A. Nielsen, M. S. Hybertsen, and L. venkataraman, ACS Nano 8, 7522 (2014).

${ }^{22}$ B. Capozzi, E. J. Dell, T. C. Berkelbach, D. R. Reichman, L. Venkataraman, and L. M. Campos, J. Am. Chem. Soc. 136, 10486 (2014).

${ }^{23}$ E. J. Dell, B. Capozzi, J. Xia, L. venkataraman, and L. M. Campos, Nat. Chem. 7, 209 (2015).

${ }^{24}$ H. Li, T. A. Su, V. Zhang, M. L. Steigerwald, C. Nuckolls, and L. Venkataraman, J. Am. Chem. Soc. 137, 5028 (2015).

${ }^{25}$ T. A. Su, H. Li, M. L. Steigerwald, L. Venkataraman, and C. Nuckolls, Nat. Chem. 7, 215 (2015).

${ }^{26}$ O. Adak, E. Rosenthal, J. Meisner, E. F. Andrade, A. N. Pasupathy, C. Nuckolls, M. S. Hybersen, and L. Venkataraman, Nano Lett. 15, 4143 (2015).

${ }^{27}$ B. Capozzi, J. Xia, O. Adak, E. J. Dell, Z. Liu, J. C. Taylor, J. B. Neaton, L. M. Campos, and L. venkataraman, Nat. Nanotechnol. 10, 522 (2015).

${ }^{28}$ T. A. Su, H. Li, V. Zhang, M. Neupane, A. Batra, R. S. Klausen, B. Kumar, M. L. Steigerwald, L. Venkataraman, and C. Nuckolls, J. Am. Chem. Soc. 137, 12400 (2015).

${ }^{29}$ M. S. Hybertsen and L. Venkataraman, Acc. Chem. Res. 49, 452 (2016).

${ }^{30}$ B. Capozzi, J. Z. Low, J. Xia, Z. Liu, J. B. Neaton, L. M. Campos, and L. Venkataraman, Nano Lett. 16, 3949 (2016).

${ }^{31}$ H. Li, M. H. Garner, Z. Shangguan, Q. Zheng, T. A. Su, M. Neupane, P. Li, A. Velian, M. L. Steigerwald, S. Xiao, C. Nuckolls, G. C. Solomon, and L. Venkataraman, Chem. Sci. 7, 5657 (2016).

${ }^{32}$ T. A. Su, H. Li, R. S. Klausen, J. R. Widawsky, A. Batra, M. L. Steigerwald, L. Venkataraman, and C. Nuckolls, J. Am. Chem. Soc. 138, 7791 (2016).

${ }^{33}$ N. T. Kim, H. Li, L. Venkataraman, and J. L. Leighton, J. Am. Chem. Soc. 138, 11505 (2016).

${ }^{34}$ Y. Q. Xue and M. A. Ratner, Phys. Rev. B 68, 115406 (2003).

${ }^{35}$ C. Li, I. Pobelov, T. Wandlowski, A. Bagrets, A. Arnold, and F. Evers, J. Am. Chem. Soc. 130, 318 (2008).

${ }^{36}$ S. Hou, J. Zhang, R. Li, J. Ning, R. Han, Z. Shen, X. Zhao, Z. Xue, and Q. Wu, Nanotechnology 16, 239 (2004).

${ }^{37}$ J. Ning, R. Li, X. Shen, Z. Qian, S. Hou, A. R. Rocha, and S. Sanvito, Nanotechnology 18, 345203 (2007).

${ }^{38}$ S. Y. Quek, M. Kamenetska, M. L. Steigerwald, H. J. Choi, S. G. Louie, M. S. Hybertsen, J. B. Neaton, and L. Venkataraman, Nat. Nanotechnol. 4, 230 (2009).

${ }^{39}$ M. Strange, C. Rostgaard, H. Hakkinen, and K. S. Thygesen, Phys. Rev. B 83, 115108 (2011).

${ }^{40}$ S. Y. Quek and K. H. Khoo, Acc. Chem. Res. 47, 3250 (2014).

${ }^{41}$ X. Y. Feng, Z. Li, and J. Yang, J. Phys. Chem. C 113, 21911 (2009).

${ }^{42}$ Y. Meir and N. S. Wingreen, Phys. Rev. Lett. 68, 2512 (1992).

${ }^{43}$ P. Hohenberg and W. Kohn, Phys. Rev. 136, B864 (1964).

${ }^{44}$ W. Kohn and L. J. Sham, Phys. Rev. 140, A1133 (1965).

${ }^{45}$ Y. Xue, S. Datta, and M. A. Ratner, Chem. Phys. 281, 151 (2002).

${ }^{46}$ M. Brandbyge, J.-L. Mozos, P. Ordejón, J. Taylor, and K. Stokbro, Phys. Rev. B 65, 165401 (2002).

${ }^{47}$ J. Zhang, S. Hou, R. Li, Z. Qian, R. Han, Z. Shen, X. Zhao, and Z. Xue, Nanotechnology 16, 3057 (2005).

${ }^{48}$ R. Li, J. Zhang, S. Hou, Z. Qian, Z. Shen, X. Zhao, and Z. Xue, Chem. Phys. 336, 127 (2007).

${ }^{49}$ A. R. Rocha, V. M. García-Suárez, S. W. Bailey, C. J. Lambert, J. Ferrer, and S. Sanvito, Nat. Mater. 4, 335 (2005).

${ }^{50}$ A. R. Rocha, V. M. García-Suárez, S. Bailey, C. Lambert, J. Ferrer, and S. Sanvito, Phys. Rev. B 73, 085414 (2006).

${ }^{51}$ J. M. Soler, E. Artacho, J. D. Gale, A. García, J. Junquera, P. Ordejón, and D. Sánchez-Portal, J. Phys.: Condens. Matter 14, 2745 (2002).

${ }^{52}$ N. Troullier and J. L. Martins, Phys. Rev. B 43, 1993 (1991).

${ }^{53}$ J. P. Perdew, K. Burke, and M. Ernzerhof, Phys. Rev. Lett. 77, 3865 (1996).

${ }^{54}$ R. Li, S. Hou, J. Zhang, Z. Qian, Z. Shen, and X. Zhao, J. Chem. Phys. 125, 194113 (2006).

${ }^{55}$ C. Toher and S. Sanvito, Phys. Rev. Lett. 99, 056801 (2007). 